\title{
Polisemia
}

\section{EL ESTUDIO DE TEXTOS EN LA FORMACIÓN FILOSÓFICA CONSIDERACIONES METODOLÓGICAS}

Carlos Miguel Gómez Rincón ${ }^{7}$

A Diego Pineda, amigo y maestro en lecturas y escrituras.

\section{Resumen:}

E I artículo sitúa en el centro de la formación filosófica, especialmente a nivel de pregrado, el estudio y la producción de textos. Arroja consideraciones metodológicas para emprender el estudio exhaustivo e integral de una obra filosófica y al respecto menciona tres tipos de lectura: el estudio intrínseco de la obra, la lectura en clave de relaciones intertextuales y la lectura en clave socio-histórica. Del primer tipo mencionado destaca la importancia del análisis, de la captación de la estructura del texto y de la interpretación de las hipótesis de lectura.

Recalca el papel del seminario como la metodología privilegiada para aprender a investigar en filosofía y subraya cómo la formación filosófica contribuye a desarrollar competencias afectivas, sociales y éticas, aparte de desarrollar destrezas cognitivas.

\section{Palabras Claves}

Filosofía, enseñanza, aprendizaje, textos filosóficos, estudio intrínseco, lectura intertextual, contexto socio-histórico, competencias afectivas.

\section{THE STUDY OF TEXTS IN THE PHILOSOPHICAL TRAINING. SOME METHODOLOGICAL CONSIDERATIONS}

\section{Abstract}

This work places studying and producing texts in the center of the philosophical formation, especially at the undergraduate level. It gives some methodological considerations for undertaking an integral and exhaustive study of a philosophical work. It mentions three types of reading: first, the intrinsic study of the work, second, the search of inter-textual relationships and third, social and historical context. In agreement with the first type, the article highlights the importance of capturing the text's structure and the hypothesis of reading.

The author emphasizes the role of the seminar like the main methodology for learning to research in philosophy. He enhances how the philosophical training 
helps to develop emotional, social and ethical skills, apart of developing cognitive skills.

\section{Key Words}

Philosophy, teaching, learning, philosophical texts, intrinsic study, inter-textual reading, social and historical context, emotional competencies.

\section{La formación filosófica}

Muchas disciplinas parecen tener claridad sobre su objeto de estudio, sus métodos, sus principios básicos, los protocolos y las técnicas de su práctica, así como sobre el tipo de productos y resultados a los que estos conducen y los criterios para evaluarlos, de modo que durante su proceso de formación los aprendices pueden tener una conciencia sobre lo que están aprendiendo y lo que van a ser capaces de hacer cuando se gradúen. A diferencia de ellas la filosofía, tal y como es "enseñada" en nuestro contexto, parece vacilar siempre a la hora de tener que aclarar estos asuntos. Esto no se debe solamente a algo así como a una deficiencia pedagógica, sino principalmente al carácter de la filosofía misma, la cual se ha autocomprendido desde sus orígenes griegos como la ciencia que se busca y nunca se encuentra del todo.

Pero desde el punto de vista de la enseñanza y el aprendizaje de la filosofía debemos intentar responder a estas cuestiones. En particular en el nivel de pregrado, ¿en qué consiste propiamente estudiar filosofía? Esto es tanto como preguntar: ¿qué es exactamente lo que se estudia?, ¿qué es lo que uno aprende?, ¿cómo se estudia eso?, ¿qué debería un estudiante ser capaz de hacer al graduarse?, y ¿cómo puede todo esto ser enseñado? Naturalmente, las respuestas a estas preguntas dependerán de la figura que uno tenga de la filosofía, es decir, del modo como comprenda la disciplina, sus preguntas centrales, sus métodos, sus fines y su historia; pero también, las respuestas variarán de acuerdo con los enfoques de los programas de estudios de filosofía. Sin embargo, la amplitud de las respuestas no puede desorientarnos al punto de hacernos perder de vista lo central, aquello que constituye en buena parte la especificidad misma del estudio filosófico. Tratemos de determinar esta especificidad atendiendo brevemente a las preguntas planteadas.

\section{A) ¿Qué es exactamente lo que se estudia en un programa de pregrado en filosofía?}

Entre las respuestas posibles a esta pregunta, que llegan inmediatamente a la mente tenemos: ideas, conceptos, problemas, autores, el Ser, lo real, el pensamiento. En cierto sentido, habría que aceptar que todos estos constituyen objetos de estudio y reflexión en la filosofía, pero a la vez parece como si no hubiéramos dicho nada. En primer lugar, porque estas posibilidades constituyen también los objetos de estudio de otras disciplinas, que de algún modo se aproximan a ellos más directamente y con una metodología precisa: la psicología o las ciencias cognitivas, por ejemplo, pueden ocuparse del estudio de las ideas, de los conceptos y del pensamiento, tomados como datos susceptibles de ser investigados mediante métodos empíricos, para determinar los procesos que los producen, sus leyes, su base neuronal, etc.

Sin embargo, uno podría decir que la filosofía estudia algo que queda fuera de las consideraciones que toman estos objetos, sólo como datos empíricos: su sentido, los principios mediante los cuales son conocidos, el lenguaje en el que se expresan, sus consecuencias éticas, etc. Pero, esto en nada resuelve el problema. Simplemente, aporta nuevos candidatos que amplían el espectro -ya de por sí en apariencia carentede-unidad y todo-comprehensivo- de objetos de estudio de la filosofía. ¡Muy bien! Entonces lo que uno estudia es una forma de pensar, algo así como un método de reflexión que sirve para considerar cualquier objeto desde una perspectiva filosófica. Pero, nuevamente, la respuesta no nos satisface plenamente, pues no existe un método 
unificado para la investigación filosófica (en efecto, cada filósofo comienza por inaugurar o reformar un método), y en la práctica, una formación filosófica no es simplemente la adquisición de una técnica o el dominio de un procedimiento, si bien existen técnicas y procedimientos importantes en el trabajo filosófico. Por lo demás, uno no aprende un método de investigación filosófica independientemente de cómo es usado por un filósofo en una obra, es decir, no aprende los pasos y principios de su aplicación para luego utilizarlos, sino que más bien estudia el método en acción, es decir, cómo es utilizado en una obra para producir pensamiento filosófico.

Entonces, ¿qué es aquello que estudiamos cuando entramos a estudiar filosofía? Alguien podría decir: ¡Un momento! Usted dijo que ciertos objetos de reflexión filosófica son estudiados también por otras disciplinas de modo más directo, pero, ¿qué ocurre con esos objetos que ni siquiera son objetos, $y$ que no son considerados por ninguna otra disciplina: el Ser, lo real, los primeros principios, etc.? Aún si abogáramos por la vocación metafísica de la filosofía, la cuestión consiste justamente en que los estudiantes no aprenden a abordar directamente estos pseudobjetos, como un biólogo aborda un sistema orgánico o un físico las leyes de la naturaleza. Más bien, lo que hacemos es estudiar interpretaciones, visiones, discursos sobre ellos, sobre el modo como pueden o no ser conocidos y sobre las implicaciones que estas interpretaciones tienen en diferentes ámbitos de la vida humana.

Esto es sumamente importante para ofrecer una respuesta medianamente satisfactoria a nuestra pregunta. En un programa de filosofía se estudian básicamente textos y son textos también lo que se produce. Puede ser que el escrito no sea el único modo de expresión o de construcción del discurso filosófico, pues bajo la forma del diálogo y de otro tipo de discusiones se establecen profundos vínculos con la oralidad; pero aún las formas orales de la filosofía son susceptibles de ser estudiadas como textos, puesto que constituyen tejidos argumentativos. Las ideas, los conceptos, los problemas, el pensamiento de los filósofos, sus discusiones, sus búsquedas y sus construcciones de sentido, llegan a nosotros a través de elaboraciones, bien sea orales o más frecuentemente escritos; además, nuestras interpretaciones, comentarios, preguntas, críticas y reflexiones también conforman textos.

Así, pues, el estudio y la producción de textos constituyen el corazón de la formación filosófica; lo que nos lleva a formular la siguiente pregunta:

\section{B) ¿Qué es lo que uno aprende cuando estudia filosofía?}

A partir de lo anterior, podemos dar una respuesta en apariencia sencilla: uno aprende a estudiar y producir textos filosóficos. ¿Nada más que esto? ¿No es esta una visión demasiado estrecha de laformación filosófica que deja por fuera dimensiones que no deberían olvidarse, como la función social de la filosofía, su tarea de pensar el presente, sus relaciones con otras disciplinas, su apertura al mundo cotidiano y a la "realidad social", su vocación pedagógica, su misión en la formación integral del ser humano, etc.? Ciertamente, ninguna de estas dimensiones ni otras semejantes deben ser dejadas de lado. Pero afirmar que el centro de la formación filosófica de pregrado consiste en aprender a estudiar y producir textos, es decir, a leer, escribir y hablar filosóficamente, ni niega la importancia de estas dimensiones ni las excluye, simplemente les asigna un escenario de trabajo y formación específico, propio del filósofo. Al hacer esto, permite comenzar a identificar con claridad las competencias y las habilidades que deben ser desarrolladas por los estudiantes. Sin esto, cualquier proyecto de formación filosófica se desvanece en buenas intenciones, se confunde con escenarios y competencias, que si bien lo pueden enriquecer no le son propios y le llevan finalmente a enredarse en una ambigüedad de concepciones. 
En efecto, la función social de la filosofía, su lugar en la construcción y transformación de la realidad, su obligación de reflexionar sobre el presente, su compromiso de dialogar con otras disciplinas académicas, con formas del saber popular y tradicional, su misión educadora y humanizadora, además de otras tareas semejantes, no pueden ser emprendidas consciente $y$ responsablemente sino a partir del estudio y de la producción de textos, que justamente por esto representan los elementos básicos de la formación filosófica. "A partir" quiere decir aquí, de un lado, "empezar por" el estudio de los textos de la tradición, sus problemas, sus conceptos; por otro, formar el propio pensamiento desde esos textos para comprender el presente y abrirse al diálogo con otros escritos, con aquellos producidos tanto por otras disciplinas como por la sabiduría popular, otras tradiciones o simplemente los discursos del sentido común. Pero también significa que el filósofo puede participar en la construcción social por medio de textos, escritos por supuesto, pero también orales cuando desempeña funciones como docente, consultor, asesor o cuando habla como filósofo con los demás.

La cuestión es que debemos comprender lo que es un texto en sentido amplio. En efecto, la tradición filosófica ofrece una gran variedad de tipos de textos y de géneros, que incluyen el diálogo, el tratado, el ensayo, el aforismo, las memorias, incluso el artículo periodístico, la poesía, el cuento y la novela. Pero además, en el ámbito de la oralidad, un discurso público, una discusión, una conversación, una clase y otras expresiones semejantes constituyen textos. Adicionalmente, las otras disciplinas y la sociedad en general generan y se constituyen en buena medida por textos de todo tipo.

Esto nos lleva a la siguiente pregunta:

\section{C) ¿Cómo se estudia un texto filosófico?}

En general, hay tres ámbitos en los que un texto cualquiera y en particular un texto filosófico puede ser estudiado, ellos suponen tres tipos de lectura que se complementan, pero deben ser aprendidos y perfeccionados con cierta independencia y en una secuencia jerárquica, pues cada uno cuenta con metodologías e intereses diferentes, pero en su orden un tipo de lectura es prerrequisito para el siguiente.

El primer tipo de lectura, necesario para los demás, se suele denominar estudio intrínseco de la obra. Como su nombre lo indica, consiste en explorar y comprender la forma como un texto está constituido: su estructura argumentativa, sus partes, las relaciones entre ellas, los mecanismos y estrategias que utiliza el autor para decir lo que dice, darle sentido a los conceptos que utiliza y crear una red de significados. Se trata aquí de abordar el texto en su interioridad, de analizarlo para descubrir la forma como funciona: no sólo lo que dice el autor, sino lo que hace para decir lo que dice. Desde esta perspectiva, leer es reconstruir las estructuras textuales que dan significado a los problemas, tesis, discusiones y conceptos de un autor: seguir el curso de producción textual del significado y poder explicarlo en sus propios términos. En este sentido, una obra filosófica se comprende como un universo autónomo que contiene en sí misma la clave de su interpretación, por lo menos en lo que se refiere al primer acceso a su sentido.

Pero, si bien toda obra es un universo autónomo, no se encuentra cerrada en sí misma, no es un universo hermético. Antes bien, sus problemas, sus conceptos, sus formas discursivas y muchos otros elementos de su estructura particular se encuentran en relación con otras obras que conforman una o varias "tradiciones". Así, el segundo tipo de lectura es el que se ocupa de investigar las relaciones intertextuales. Semejante investigación puede revestir distintas formas. Por ejemplo, puede interesarse por rastrear la génesis de los problemas y conceptos de un texto en otros textos, ya sea escritos por el mismo autor o por otros. Desde esta perspectiva, la comprensión del 
sentido de una obra exige la reconstrucción de un horizonte de significados más amplio, constituido por las obras leídas y discutidas por el autor, por aquellas que influyeron en él o contra las cuales discute. Muchas veces este tipo de relaciones intertextuales están sugeridas en la obra misma que se está estudiando, mediante referencias, reconocimientos y críticas directas a otras obras y autores citados con nombre propio. En otros casos, descubrirlas es una tarea ardua que demanda recurrir a bibliografía secundaria y a comentarios sobre la obra estudiada.

En todo caso, este tipo de lectura permite captar el desarrollo de los conceptos y problemas de un autor, bien sea a lo largo de un conjunto de sus obras o en relación con otras. Esto permite comprender cómo se construyen los significados de los conceptos a partir de la discusión con otros y de la reflexión sobre significados anteriores, en relación con el contexto de una tradición de problemas, conceptos y modos de investigación. Además, en este tipo de lectura se abre un espacio para el trabajo interdisciplinario, pues en la tradición filosófica con mucha frecuencia se elaboran los textos dialogando con otras ciencias y disciplinas cuyos principios deben ser estudiados para la comprensión del texto en cuestión. Incluso, más allá de esto, una lectura de este tipo permite establecer relaciones entre las distintas disciplinas y la filosofía; por ejemplo, al captar la influencia de una obra filosófica en cierta teoría científica o ideario político, pero también cuando se indagan los principios filosóficos que subyacen a las obras de otras disciplinas.

Finalmente, un texto no sólo forma parte de una tradición de textos, sino que seencuentra en relación con un contexto aún mucho más amplio, el que suponen la sociedad y la historia, con el cual se vincula de múltiples maneras. Así, el tercer tipo de lectura es el que se realiza en clave socio-histórica. De manera semejante a lo que ocurre con el segundo tipo de lectura, el estudio de textos desde esta perspectiva puede tomar varias formas, utilizar distintas metodologías y buscar diversos fines, dependiendo del ámbito de la relación texto-sociedad que pretenda investigar. Por ejemplo, puede tratarse de comprender la situación histórico cultural del autor en el momento de elaboración de la obra, para mostrar hasta qué punto esta es un "producto de su tiempo" e inclusive, desde un punto de vista psicológico, un "producto de las condiciones personales del autor". El contexto aquí se asume como causa de la obra, por tanto, el sentido de esta no puede desligarse del conjunto de fuerzas históricas, sociales y políticas que contribuyeron a su producción. Desde otro punto de vista, el contexto puede comprenderse también como aquello sobre lo cual la obra reflexiona, y entonces se trata de estudiarlo para comprender las referencias, las críticas y las propuestas que sobre él realiza el texto.

Pero estos no son los únicos tipos de lectura en clave socio-histórica. Puede también buscarse comprender no el contexto social en el que la obra fue pensada, sino el contexto en el que es leída. Esto es tanto como rastrear las interpretaciones que se han hecho del texto en cuestión y el modo como estos han contribuido en la construcción de la sociedad, la comprensión de la realidad y el surgimiento de nuevas prácticas, saberes y disciplinas. O, también, la lectura puede ocuparse de repensar los problemas y conceptos de un texto en relación con otros contextos, identificar sus aportes, sus límites, sus posibilidades, en pocas palabras, discutir con el texto produciendo un nuevo texto con algo de originalidad y pensamiento propio, frente a los problemas y necesidades del lector.

En el siguiente gráfico podemos observar de manera esquemática el modo como estos tres tipos de lectura surgen a partir del ámbito de relaciones textuales que pretenden investigar: 


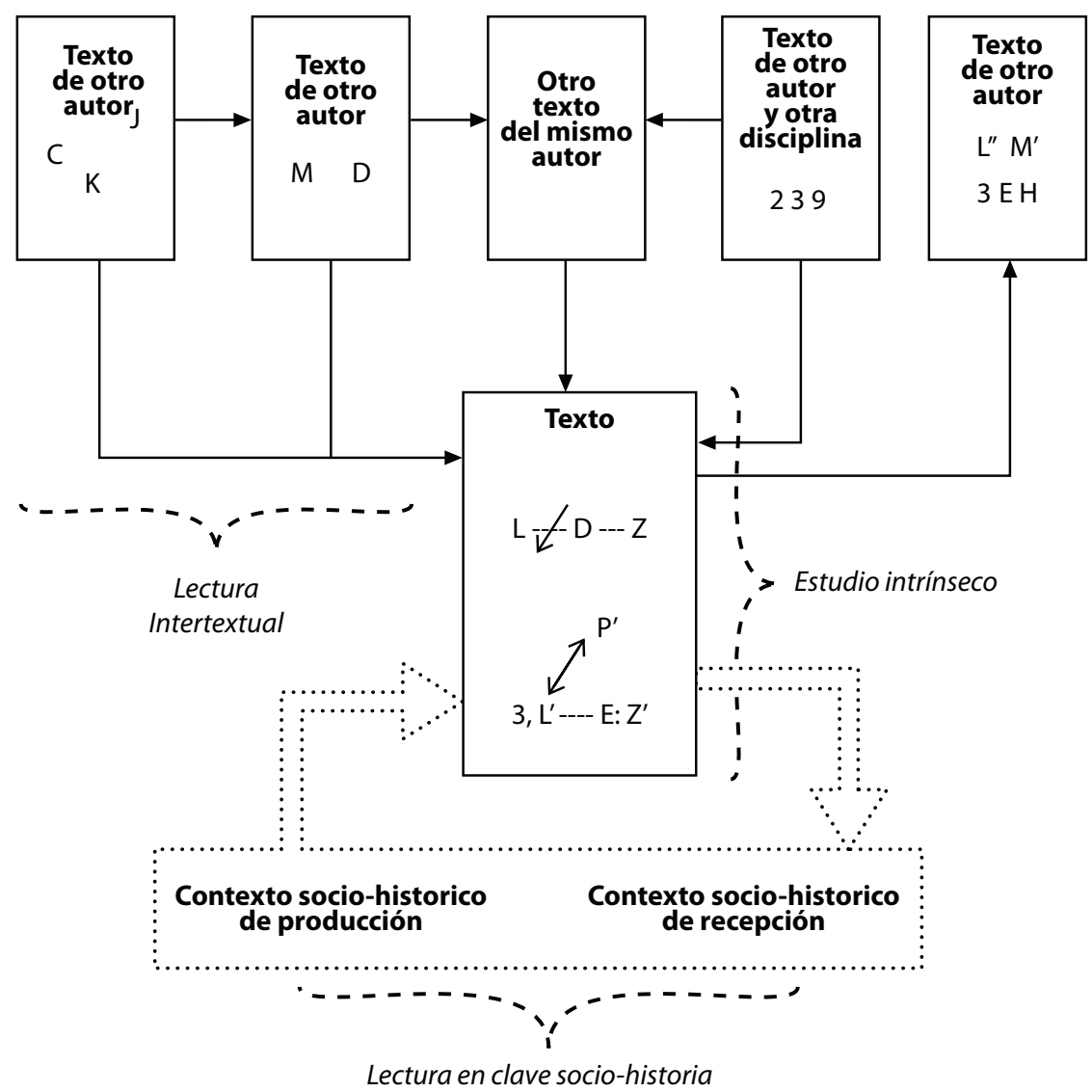

Evidentemente, es deseable buscar la articulación de estos tres tipos de lectura ${ }^{2}$ para el estudio exhaustivo e integral de una obra filosófica. No obstante, pretender que esto deba y pueda hacerse siempre, sobre todo en pregrado, no es sólo demasiado pretencioso sino poco pedagógico. En tanto eje fundamental de la formación filosófica, la lectura filosófica es ante todo una forma de investigación cuyos métodos e intenciones dependen de cuál de los tipos de lectura mencionados -inclusive cuál de sus subtipos o enfoques específicos- se pretenda realizar. La articulación de métodos e intereses diversos no puede ser llevada a cabo sino cuando se domina cada uno por separado. Alcanzar el domino de uno o varios tipos de lectura debe ser, por consiguiente, uno de los objetivos principales de la formación filosófica.

Con esto podemos abordar la cuarta pregunta:

\section{D) ¿Qué debe saber hacer un graduado de un programa de filosofía?}

¡Leer bien! Esto quiere decir, ser capaz de estudiar sistemáticamente un texto o un grupo de textos desde cualquiera de las perspectivas anteriores, dependiendo del tipo de orientación del programa en cuestión y de los intereses del estudiante. Pero, en contra de otros tipos de lectura, la lectura filosófica se encuentra muy lejos de ser un ejercicio pasivo; se trata de una actividad productiva cuyos resultados deben ser siempre visibles, contrastables y evaluables. Toda lectura filosófica exige la producción de otros textos. Consecuentemente, el resultado de la lectura filosófica no es algo parte tomamos lo que hemos dicho aquí. Así, por ejemplo, el estructuralismo y la semiótica ofrecen modelos claves para el estudio intrínseco; la socio-crítica y la psico-crítica, por un lado, y por otro, la estética de la recepción, representan modos de investigación textual en clave socio-histórica; y la literatura comparada es un caso de lectura inter-textual. Para una introducción al panorama completo de las teorías literarias del siglo XX, Cf. Cuesta Abad, J. M. y Jiménez Heffernan, J. (Eds.) (2005). Teorías Literarias del siglo XX, Madrid: Akal. 
así como la sola "comprensión mental" de los significados de un texto que luego pueden ser repetidos por el lector. Más bien, consiste en una serie de ejercicios de producción de nuevos textos que van desde la elaboración de esquemas conceptuales hasta la escritura de monografías y comentarios, incluso, hasta la elaboración de ensayos y escritos de investigación original. No obstante, esto último no se espera del graduado de un programa de pregado, más bien de un magíster y de un doctor en filosofía. Para el primero, la capacidad de realizar trabajos de tipo monográfico y exegético constituye una exigencia suficiente. Por lo demás, la capacidad de producir esta clase de textos implica también la habilidad de comunicarse oralmente, participar en discusiones, realizar presentaciones y dirigir investigaciones y procesos de reflexión en este ámbito.

Así, saber leer, escribir y hablar filosóficamente son las metas formativas de un estudiante de filosofía en el pregrado. Lo que por supuesto, no le asigna a estas tareas un ámbito profesional único e inamovible, ni un rol social cerrado y limitado a la academia, pues la lectura, la escritura y la comunicación filosóficas pueden encontrar lugar en una gran variedad de escenarios y procesos profesionales y sociales, algunas opciones son la investigación y planeación pedagógicas, los medios de comunicación, el derecho, la administración de empresas, la resolución de conflictos, la asesoría, la consultaría personal y grupal, la gestión, el diseño y la conducción de proyectos sociales. Pero, ciertamente, las posibilidades de que estas tareas propias del ejercicio filosófico encuentren y creen espacios de participación diferentes a la academia, dependen de qué tan bien un profesional sepa llevarlas a cabo.

Quisiera subrayar esta idea para combatir un riesgo que surge cuando aparece la cuestión del compromiso social de la filosofía y del interés por asignarle una misión más amplia que la de los estrechos muros de las academias. Sin duda, estas preocupaciones no sólo responden a una exigencia ética, sino que resultan del todo legítimas en tanto asumen a la filosofía como una disciplina viva, en construcción, que tiene algo que decir y aportar al presente. Pero atender a ellas no puede significar una pérdida de la especificidad de la disciplina filosófica, confundiéndola con otras o asignándole tareas que sobrepasan sus límites. Solamente habiendo comprendido en qué consiste el ejercicio filosófico y la maestría en las practicas de lectura, escritura y comunicación que lo conforman, puede un graduado de filosofía desempeñarse como tal en otros escenarios.

Finalmente, hemos de tratar la última pregunta:

\section{E) ¿Cómo puede enseñarse y aprenderse a leer, escribir y expresarse filosóficamente?}

Si bien los currículos tradicionales de filosofía otorgan, más o menos conscientemente, a cada tipo de lectura un espacio de desarrollo particular (i.e. las clases de historia de la filosofía para la lectura en clave sociohistórica, las asignaturas sistemáticas para la lectura intertextual y los seminarios para el estudio intrínseco), no es exagerado afirmar que todas las metodologías de la enseñanza y el estudio de la filosofía, al igual que todos los tipos de lectura filosófica, exigen y parten del estudio intrínseco de las obras. En efecto, el primer paso en una lectura intertextual, tanto como en otra lectura hecha en clave socio-histórica, es la comprensión de las estructuras argumentativas, los problemas, los conceptos y las estrategias discursivas de una o varias obras (filosóficas y de otras disciplinas). Sin esto, difícilmente pueden establecerse relaciones con la tradición, con otras disciplinas, con el contexto de producción o con el de recepción, pues simplemente no se conoce ni se comprende a profundidad aquello con lo cual se están tratando de establecer las relaciones.

En este sentido, el estudio de la interioridad de las obras y la capacidad para captar y reconstruir el modo como funcionan es la condición previa para toda lectura 
filosófica. Por eso, merece ser aprendido y perfeccionado con especial dedicación, reforzando esta idea el hecho de que una buena escritura, al igual que una buena capacidad expresiva, dependen también de la habilidad para identificar, generar, contrastar y evaluar conscientemente estructuras argumentativas ordenadas, coherentes y comprehensivas.

Consecuentemente, responder a la pregunta de este apartado implica, ante todo, explicar cómo se realiza el estudio intrínseco de una obra filosófica, es decir, en qué consiste leer y producir textos desde esta perspectiva. Pero como la pregunta también involucra el cómo aprender y enseñar esta tarea, nuestra respuesta debe explicar la metodología del estudio intrínseco en el contexto de la práctica pedagógica en la que suele enseñarse en las escuelas de filosofía, justamente aquella práctica que no sin razón se considera el ejercicio básico de la formación filosófica: el seminario.

Desafortunadamente, no podremos profundizar aquí en lo otros tipos de lectura y sólo nos referiremos a ellos en tanto que son ocasionalmente utilizados para enriquecer el trabajo del seminario. Sus metodologías son tan variadas y complejas, que para su presentación requieren de un volumen completo ${ }^{3}$. Por ahora, nos contentaremos si logramos ofrecer a los estudiantes de filosofía una orientación metodológica que pueda servirles para aprender a leer mejor una obra filosófica en el contexto del seminario.

Una aclaración es aquí necesaria. Lo que sigue, como toda consideración metodológica, es solamente la presentación de un punto de vista desde el cual se pretende abordar el estudio de un texto, de manera sistemática y conciente. Por eso, no se trata de algo así como del canon del estudio intrínseco de obras filosóficas (o del seminario). Otros puntos de vista metodológicos y perspectivas de acceso a la obra dentro del enfoque general del estudio intrínseco -que es el que consideraremos aquí- son bienvenidos como alternativas que pueden enriquecer la lectura analítica y estructural que propondremos (tanto como nuestra versión del seminario). Por lo demás, una lectura filosófica no sería tal si no se encontrara abierta a repensar constantemente los principios que la orientan y a discutirlos con otros.

\section{El seminario en la formación filosófica}

El seminario (alemán) ${ }^{4}$ es una metodología de la enseñanza y del estudio propio de la educación superior, que se basa en el trabajo comunitario y autónomo de un grupo de estudiantes que, con la dirección de un profesor, se reúnen para la lectura detenida y rigurosa de una o varias obras (o fragmentos) de uno o varios autores (que el tema central del seminario relaciona). Se trata fundamentalmente de un ejercicio de lectura intrínseca dirigida, en la que cada participante debe desempeñar, cuando le corresponda, uno de los siguientes tres roles principales:

- Expositor (presenta el texto asignado para la sesión).

- Protocolante (recoge la discusión).

- Interlocutor (participa en la discusión).

Adicionalmente, el rol del director suele ser desempaño por el profesor, cuya función, a diferencia de lo que ocurre en las cátedras y clases magistrales, no consiste en transmitir una serie de contenidos e informaciones sobre los textos y autores en cuestión, sino en guiar el proceso de lectura, aportando los elementos metodológicos y conceptuales necesarios para esto, tanto como motivando 
la discusión mediante preguntas, el ofrecimiento de otras posibilidades de interpretación de los textos, el señalamiento de inconsistencias, vacíos y contradicciones en las interpretaciones ofrecidas, y otras operaciones semejantes. Por lo demás, el director suele, cuando es necesario para la comprensión del texto, recurrir a herramientas y productos de la lectura intertextual o en clave socio-histórica. En algunos casos, se incluye otro rol, el del correlator, quien tiene a su cargo la tarea de cotejar, evaluar, complementar y corregir al expositor. Pero, en general, este rol puede ser asumido por el resto del grupo, quienes deben participar como interlocutores en la discusión sobre la interpretación del texto.

En la formación filosófica, el seminario es el lugar por excelencia para aprender a investigar. En el nivel de pregrado, esto significa, como hemos dicho, aprender a estudiar y producir textos desde una perspectiva exegética y monográfica. Si bien hay distintos tipos y propósitos para el seminario, la exégesis y la investigación monográfica, en tanto que son el resultado de la lectura intrínseca de una obra, son la base y el punto de partida para los demás. Aquí sólo nos ocuparemos de este tipo de seminario que constituye, a nuestro modo de ver, el más adecuado para la formación filosófica de pregrado.

Estructuralmente una sesión de seminario cuenta con cuatro momentos:

- Preparación (lectura personal del texto de acuerdo con las pautas del estudio intrínseco de la obra).

- Lectura del protocolo anterior (que permite revisar el estado de comprensión del texto a partir de la sesión anterior y ubicarse en las cuestiones y momentos del texto en el se encuentra la lectura).

- Exposición (que presenta la sección del texto asignada para la sesión y abre las líneas de discusión).
- Discusión (sobre las interpretaciones del texto que presenta la exposición, sus falencias, vacíos y aportes, tanto como sobre preguntas que surjan a partir de ella y enriquezcan la comprensión de los problemas, conceptos y discusiones del texto. Todo esto es lo que tiene que ser recogido en el protocolo de la sesión).

El adecuado desempeño de los roles del seminario y el éxito en la realización de sus momentos dependen de que cada uno de sus participantes se comprometa con los dos ejercicios fundamentales de la formación filosófica: la lectura (en este caso intrínseca) y la producción de textos (orales y escritos). Consideremos las pautas metodológicas y los criterios de realización de la lectura intrínseca, los cuales establecen también la base para una buena escritura.

\subsection{El estudio intrínseco de una obra filosófica}

El estudio intrínseco de una obra filosófica tiene tres características principales: es una lectura analítica, pretende captar y exponer la estructura de un texto y asume que el sentido de una obra se construye mediante la formulación, contrastación y evaluación de hipótesis de lectura por parte del lector. Estas características, antes que tres pasos metodológicos, son operaciones complementarias que se interrelacionan en todo momento y que implican el desarrollo de una gran cantidad de habilidades cognitivas, tanto como el cultivo de una sensibilidad que bien podemos denominar literaria ${ }^{5}$. La lectura personal para preparar el seminario y, por supuesto, la exposición, deben ante todo cumplir los requisitos de esta forma de investigación.

¿Cómo realizar una lectura analítica, estructural e interpretativa? A continuación presentamos, de modo esquemático, algunos principios y pautas básicas que pueden ayudarnos en esta tarea. 


\subsubsection{Principios del estudio intrínseco}

Primero: Comprender una obra es mucho más que ser capaz de responder a la pregunta: ¿qué dice el autor? Tener presente este principio es fundamental para una buena lectura filosófica. No se trata de captar "las ideas principales" de una obra, sus conclusiones o tesis centrales. Pues, iqué es lo que llamamos una "idea principal"?, ¿qué criterios podemos utilizar para determinar esto? Generalmente, los estudiantes confunden las ideas principales con las únicas que lograron entender, y como resultado sus exposiciones son un reguero de frases desconectadas incapaces de explicar el sentido del texto.

Comprender un texto tampoco es "sintetizarlo" o "resumirlo". Esta operación se basa en la anterior y por lo tanto comete los mismos errores: los textos filosóficos no pueden ser resumidos sin violentarlos, sin perder su dimensión fundamental, que comprende la discusión, la argumentación, la construcción dialógica de conceptos en el tratamiento de un problema. Esta dimensión simplemente no corresponde a lo que el autor dice, sino más bien a lo que hace en el texto: ¿cómo lo dice?, ¿en qué momento lo dice?, ¿por qué lo dice en esta parte y no en otra?, ¿para qué lo dice?, ¿contra quién lo dice?, ¿qué recursos argumentativos utiliza para decirlo?, ¿en qué relación está lo que dice con lo dicho anteriormente y con el plan general de la obra?, ¿el autor busca producir algún efecto con lo que dice?, ¿cuál es su tono?, etc. Si en una exposición de seminario no se abordan preguntas de este estilo, simplemente no podemos afirmar que estemos comprendiendo un texto.

Finalmente, comprender un texto no es interrogarlo descuidadamente con preguntas, críticas y relaciones que le resultan completamente exteriores. Kant se equivocó porque no tuvo en cuenta la genética, el concepto de yo de Descartes es falso porque la psicología actual dice otra cosa, Platón ya fue superado por Nietzsche, las ideas de Leibniz sobre la sustancia carecen de una opción ético-política, etc. Quien no consigue entrar en el mundo propio de un texto, habitar en su horizonte de significados y percibir desde su perspectiva, es incapaz de comprenderlo. Además de lo dicho anteriormente, esto implica ser capaz de captar y rastrear el modo como sus ideas, problemas y conceptos adquieren significado dentro del texto, un significado que no tenían antes ni tienen en otros textos que utilicen las mismas palabras. ${ }^{6}$ Cada obra produce el significado de sus conceptos y problemas a partir del modo como estos se relacionan entre sí dentro del texto. ¿Qué está implícito en esta tesis?, ¿qué consecuencias tiene para lo dicho antes?, ¿sobre cuál de las ideas anteriores se basa?, ¿qué elementos nuevos y matices aporta para la comprensión del problema o el concepto que está desarrollando?, ¿se contradice esto con lo anterior?, ¿cómo cambia el sentido?, ¿significan lo mismo este concepto que aquel otro?, ¿en qué se diferencian estas dos nociones y por qué es importante la distinción?, etc. Preguntas de este tipo orientan nuestra lectura hacia la comprensión de las relaciones entre los elementos constitutivos de un texto y el modo como se van construyendo sus significados en el proceso argumentativo.

Así, el sentido de un texto depende por lo menos de tres dimensiones: a) la de sus contenidos conceptuales (qué dice); b) la de sus formas y procedimientos argumentativos (qué hace para decir); y c) la de las relaciones intratextuales mediante los cuales produce el significado de sus conceptos (cómo va apareciendo lo dicho).

Segundo: Un texto es un tejido, o mejor, un organismo vivo, y como tal tiene una estructura, esto es, un conjunto de partes que desempeñan funciones diferentes en relación con el todo, ninguna de las cuales existe fuera de esta relación. Leer filosóficamente es ser capaz de captar y 
explicar la estructura de un texto. Esto implica un conocimiento cada vez más exacto sobre el modo de ser de la filosofía, en tanto es un tipo de discurso con características, elementos constitutivos, formas y acciones lingüísticas, géneros y dimensiones propias y diferentes de otros discursos.

Este conocimiento es justamente lo que nos permite responder a cuestiones como: ¿qué hace que un texto sea filosófico?, ¿qué lo diferencia de otros textos?, ¿cómo se hace la filosofía?, ¿en qué consiste pensar filosóficamente?, ¿cuáles son las herramientas de la investigación filosófica y por qué es diferente de otros tipos de investigación?, etc. En consecuencia, se trata de un conocimiento indispensable e ineludible para un estudiante de filosofía. ¡Y se adquiere en la práctica de la lectura estructural!

Para realizar una lectura estructural pueden tenerse en cuenta las siguientes sugerencias:

\section{1)Reconocer el género del texto}

Este es el primer paso para comprender que el sentido de un texto filosófico se construye mediante una lectura que articule las tres dimensiones antes indicadas (lo que se dice, lo que se hace y la manera como aparecen los significados) y consiste en combatir un viejo prejuicio bastante arraigado en algunos lectores: la idea según la cual la forma de una obra no tiene nada que ver con su contenido, es decir, que lo que el autor quiere decir es independiente del tipo de discurso, de las estrategias expresivas $y$, en pocas palabras, de la clase de texto que decida escribir. De acuerdo con esta idea, la forma de un texto es sólo un recipiente neutral donde se deposita el contenido que es justamente aquello que debe ser comprendido.' Esta presuposición no sólo es falsa sino constituye un gran obstáculo para la lectura. Por el contrario, igual que para las obras literarias, forma y contenido son inseparables: el hecho que un autor haya escogido expresar sus ideas mediante un poema, un diálogo, un aforismo o un tratado es ya un significado de la obra. ¿En qué relación se encuentran lo dicho con la forma como se dice?

El género de una obra implica tanto una actitud como una decisión del autor, las cuales determinan en buena parte los recursos argumentativos y expresivos que se utilizan para pensar filosóficamente. Esto es sumamente importante para la comprensión del modo de ser de la filosofía. Un texto filosófico es mucho más que un medio de presentación de las ideas de un autor. Es sobre todo un medio de producción de las ideas, es decir, no una vasija para depositar las ideas ya pensadas para luego transmitirlas, sino, en sí mismo, un camino de constitución del pensamiento. La linealidad del texto, sus ires y venires, sus niveles y momentos son los de la reflexión. Sólo por eso se trata de una obra: en su elaboración se juega el curso del pensamiento mismo, que no puede ser pensado nunca plenamente antes e independientemente del texto. La obra no existía en la mente del autor antes de ser escrita, o por lo menos no como la conocemos $y$, en ese sentido, la escritura no consiste en contar lo pensado, sino en pensarlo contándolo.

Todo esto nos indica ya que la sensibilidad ante el texto, la apreciación de su materialidad, el reconocimiento de su forma particular de ser, son indispensables para leer bien, pues es la materialidad concreta de una obra lo que puede ser estudiado.

\section{2) Determinar las partes principales de un texto}

Como todo organismo, un texto se compone de partes relacionadas entre sí. Por eso, leer bien exige poder dar cuenta de las partes de un texto, las cuales muchas veces dependen de sus divisiones internas, indicadas, por ejemplo mediante subtítulos, parágrafos y numerales. Pero, generalmente 
en los textos filosóficos, esos componentes pueden identificarse mejor a partir de los temas y problemas que aborda el autor. Esto quiere decir que las partes de un texto filosófico no corresponden siempre, directa y explícitamente, a las divisiones visibles efectuadas por el autor. Por eso, determinar las partes de un texto es un proceso que demanda tanto creatividad por parte del lector, como la elección de un criterio. Así, por ejemplo, uno puede dividir un texto en partes a partir de un criterio temáticoproblemático: desde este párrafo hasta este, el autor se ocupa de tal pregunta, desarrolla este concepto, presenta esta discusión, etc.

Esto, por supuesto, implica leer entre líneas para captar cómo se construye el texto en las tres dimensiones indicadas. Vale decir: esta parte se diferencia de la siguiente por su tema, por ejemplo, el concepto del Bien, (qué dice); pero también por las formas argumentativas que el autor utiliza para desarrollarlo, por ejemplo, discute con la posición de otros filósofos refutando las premisas sobre las que se basaron sus conclusiones y ofreciendo algunos casos que ilustren su posición (qué hace); finalmente esta parte aporta elementos que enriquecen el modo en que el concepto había sido definido anteriormente, deja abiertas tales cuestiones que serán tratadas luego, implica tal tesis que el autor no plantea explícitamente, diferencia entre dos nociones contrarias sobre lo Bueno, etc., (cómo va apareciendo lo dicho).

3) Identificar las partes subordinadas y los elementos que conforman una parte principal

Cada una de las partes de un texto se compone de subpartes, las cuales a su vez están constituidas por elementos; del mismo modo que un cuerpo se compone de sistemas, ellos de órganos y estos de tipos de tejidos, etc. Por eso, una lectura estructural juiciosa es delicadamente analítica, esto es, capaz de rastrear el texto hasta en sus más pequeños detalles, operaciones y matices. Lograr esto implica preguntarse: ¿cuál es la unidad mínima de significado de un discurso filosófico?
A diferencia de un cuerpo, cuyas estructuras son fijas $y$, por decirlo de algún modo, universales (por ejemplo, se afirma que la célula es la unidad mínima constitutiva de todos los cuerpos orgánicos), la unidad mínima de significado de un texto filosófico no puede identificarse fácilmente con uno sólo de sus elementos. Decir, por ejemplo, que el concepto es la unidad mínima de significado de todos los textos filosóficos es problemático, al menos por dos razones: 1) porque semejante afirmación pone el énfasis sobre la dimensión de lo dicho, descuidando las otras dos; 2) porque el significado de un concepto no es nunca autoevidente, sino que se construye argumentativamente. Así, por ejemplo, el significado del concepto de bien, depende de las relaciones con otros conceptos y varía entre una estructura argumentativa y otra.

Así, dado que el significado de un término es siempre contextual, hemos de suponer más bien que la unidad mínima de significado de un texto filosófico es una estructura argumentativa: un argumento en sentido amplio (y no sólo entendido como la demostración de una proposición a partir de otras), o mejor, distintos tipos de formas argumentativas. Estas son los elementos que componen las subpartes de un texto. Si bien realizar una tipología de estas formas argumentativas puede resultar útil, rebasa la intención de este artículo. Para nosotros es suficiente con decir que los estudiantes de filosofía deben aprender a identificarlas captando el propósito y la función que cumplen en el texto. Algunos ejemplos de estas formas incluyen tipos de razonamiento formal como el silogismo, pero también de otras formas de razonamiento como la analogía, y aún de operaciones no necesariamente destinadas a demostrar una conclusión como el análisis de casos y proposiciones, la identificación de supuestos, la definición, la explicación de las preguntas, la comparación, la ejemplificación, etc.

Adicionalmente, así como el significado de un término depende del modo como es usado en el argumento y de las relaciones 
que establece con otros términos, el sentido del argumento depende de la relación que estable con una estructura argumentativa mayor a la cual responde, por ejemplo la del problema general que está siendo tratado. Esto quiere decir que el sentido de cada uno de los elementos del texto es relacional, depende de otros elementos $y$, en general, del todo.

El siguiente esquema intenta representar la estructura de un capítulo imaginario de un texto filosófico. En él puede visualizarse la dependencia recíproca entre las partes, cuya relación determina el sentido de cada una:

\section{Capítulo 1}

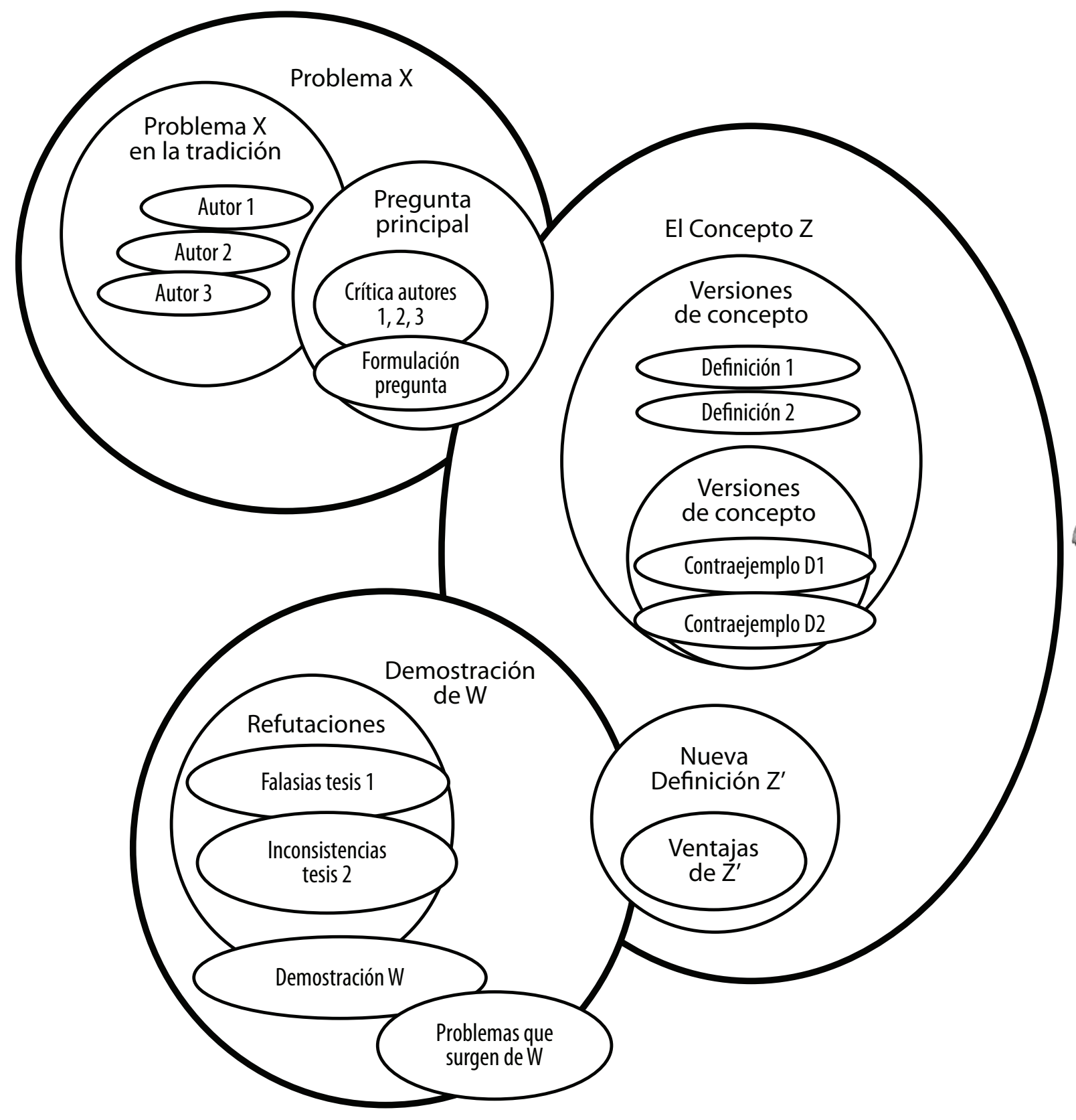


4) Exponer las relaciones entre las partes:

¿Cómo se vinculan un elemento con otro para formar una subparte?, ¿cómo se relacionan las subpartes con las partes y estas con el todo? En consecuencia con lo que hemos dicho hasta ahora, establecer las relaciones entre las partes y los elementos que constituyen el texto es fundamental para comprender su sentido. $Y$, del mismo modo que no hay un elemento mínimo único del que estén hechos todos los textos filosóficos, tampoco existe una sola forma de relación entre las partes. ¡No hay una estructura única, común a todos los textos! En efecto, esas relaciones pueden ser de tipo lógicoargumentativo: implicación, consecuencia, identidad, divergencia, causalidad, etc.; y también de tipo metodológico, en tanto cada parte sea un momento y efectúe un paso del método, ocupe un lugar en el sistema, responda al plan de la obra, etc.

No podemos aquí ni indicar todos los tipos de relaciones posibles que pueden encontrarse en un texto ni señalar todas las habilidades cognitivas que deben desarrollar los estudiantes para ello. Pero hemos de notar que una buena formación en lógica contribuye mucho a generar hábitos de lectura cuidadosa, en particular, cuando se enfoca hacia la identificación, el análisis, la diagramación y la evaluación de estructuras argumentativas tanto formales como no formales.

Debemos enfatizar que captar las relaciones entre las partes y los elementos es propiamente comprender un texto, pues implica articular las dimensiones de lo que el texto dice, lo que hace para decir y el modo como aparece el significado. Cada una depende del resto y de su interacción surge el sentido del texto. Por eso, una lectura analítica y estructural no pude descuidar ningún fragmento, por poco importante que parezca. En realidad, la costumbre de "saltarse" párrafos y pies de página, tan común en los estudiantes, indica justamente que no han adquirido maestría en la lectura. Aún si no se comprende una sola frase, el estudiante debe ser honesto y presentar en la exposición su dificultad en lugar de ocultarla, pues de ese elemento puede depender la comprensión del resto. "Dar cuenta de cada párrafo", tal debe ser el lema de este tipo de lectura.

\section{5) Rastrear relaciones transversales:}

A diferencia del lenguaje pictórico, en el que todo es simultáneo, el lenguaje verbal de los textos filosóficos es sucesivo. Esto quiere decir que se desarrolla en el tiempo (actualizado en la lectura). La argumentación se despliega a lo largo de la obra, y nosotros la vamos siguiendo como pasajeros de un viaje: en el camino, no todo está presente a la vez desde el principio hasta el final. Así, al igual que los personajes de una novela, los problemas y conceptos aparecen una y otra vez en distintas partes. Pero ninguna de ellas por sí sola es suficiente para dar cuenta de su significado. Es necesario rastrear lo que cada aparición de un concepto o un problema aporta para su comprensión.

Esto hace de la lectura una forma de investigación, que consiste en construir el significado de los elementos del texto, mediante el seguimiento de sus sucesivas apariciones a lo largo de una obra, comparándolas y contrastándolas hasta lograr la imagen más amplia posible de la cuestión. Un trabajo final de seminario, por ejemplo, puede consistir justamente en una investigación de este tipo: la exploración y la presentación de un concepto o problema a lo largo de una o varias obras.

6) Integrar las partes y sus relaciones presentando la estructura textual mediante la elaboración de esquemas:

Finalmente, es indispensable integrar todas las operaciones anteriores en un esquema que reconstruya la estructura del texto leído. La elaboración de semejante esquema es el primer producto de la lectura intrínseca de una obra, sirve como base para la posterior redacción de la exposición, para ordenar y clarificar el protocolo y, al final del seminario, 
cuando se cuenta con una serie de estos esquemas que dan cuenta de la totalidad de la obra estudiada en el seminario, permite rastrear el problema que se desea investigar en el trabajo final. Por lo tanto, no realizarlo es tanto como no haber leído, o como leer en vano, pues al final simplemente no se cuenta con nada. Por lo demás, la elaboración de un esquema facilita la comprensión de lo leído al representarlo y ayuda a determinar sus partes y las relaciones entre ellas.

No existe un modelo único para la realización de estos esquemas y lo deseable es que cada estudiante genere su propia técnica, inventando convenciones, nomenclaturas y modos de indicar los diversos tipos de relaciones entre las partes. En cualquier caso, un buen esquema debe por lo menos: 1) presentar todas las partes y subpartes, 2) ordenarlas de acuerdo a la estructura del texto, 3) señalar relaciones transversales, 4) presentar cada una de las formas argumentativas utilizadas, 6) permitir ver su función en el texto, 7) incluir anotaciones personales, preguntas, comentarios y relaciones. Así, un buen esquema es una especie de presentación de la anatomía del texto, que por lo tanto, debe dar cuenta de todo el texto (¡párrafo por párrafo!).

Una estrategia que puede ayudar a realizar buenos esquemas consiste en organizar las partes y subpartes utilizando algún tipo de nomenclatura que permita ver su lugar en el todo y seguir el curso de la argumentación. Como ejemplo ofrecemos el siguiente cuadro, que representa un esquema de lectura a partir del capítulo imaginario cuya estructura presentamos arriba. Por supuesto, no se trata de un esquema completo puesto que no presenta cada forma argumentativa, no señala relaciones transversales ni incluye comentarios personales. Pero, representa un modo efectivo de organizar y presentar los elementos del texto, lo que aquí aparece más bien como títulos y subtítulos, lo cual puede ser complementado incluyendo todo lo demás.

\section{Título del texto \\ (Capítulo 1)}

1. Primera parte:

1.1. Presentación del problema X

1.1.1.Contexto del problema en la tradición:

a) autor 1

b) autor 2

c) autor 3

1.2. Pregunta principal

a) crítica a los autores de la tradición.

b) formulación y explicación de la nueva manera de comprender la pregunta.

2. Segunda parte:

2.1. Presentación del concepto Z.

2.1.1. Formas como ha sido entendido el concepto:

a) definición 1

b) definición 2

2.1.2. Problemas de las definiciones anteriores

a) contraejemplos definición 1 .

b) demostración de la contradicción de definición 2 .

2.2. nueva definición propuesta: $z$.

2.2.1. Ventajas que aporta la nueva definición para la comprensión del problema.

3. Tercera parte:

3.1. Argumento central para demostrar W

3.1.1. Refutación de tesis de otros autores

a) Presentación y demostración de las falacias de tesis 1 .

b) Presentación y demostración de la inconsistencia de tesis

2.

3.1.2. Demostración de W.

3.2. Nuevos problemas que surgen a partir de W.

Por lo demás, la dificultad que experimentan muchos estudiantes a la hora de escribir puede ser en gran medida solucionada mediante la realización de un esquema del texto que se pretende realizar. Antes de redactar la exposición, el protocolo o un trabajo cualquiera es de gran ayuda organizar las ideas en un mapa conceptual de cualquier tipo, de modo que al escribir se sepa más o menos con claridad qué se quiere decir y hacer en cada parte del texto.

Para facilitar el trabajo de escritura, se pueden indicar en el esquema, por ejemplo, las referencias y las citas textuales que se realizarán, los comentarios y las preguntas personales, así como las pistas para resolverlas. De este modo, el esquema se convierte en una especie de carta de 
navegación, de mapa de la escritura, la cual, no obstante puede cambiar el esquema cuando sea necesario y complementarlo con las nuevas ideas, intuiciones y comprensiones que surgen en el proceso mismo de escribir.

Además, planear los textos ayuda a generar conciencia de la propia escritura y a desarrollar la destreza de crear y utilizar estructuras argumentativas coherentes y claras. Del mismo modo que todo texto que leemos es una estructura, cada texto que producimos debe también serlo. Aquí se pude ver con toda claridad qué significa aquella frase cotidiana según la cual "escribir es darle forma al pensamiento", y aún podríamos ampliarla diciendo que la lectura y la escritura filosóficas son un ejercicio de arquitectura del pensamiento. De donde, en buena parte, deriva el muchas veces olvidado gozo estético de la filosofía.

Tercero: El sentido de un texto seconstruyeen la relación con el lector. Fuera deesta relación, los conceptos, las formas argumentativas, las partes y sus interrelaciones, propiamente hablando no tienen sentido. Por eso, la lectura es un proceso creativo en todo el sentido de la palabra: leer bien es tanto como reescribir la obra ${ }^{8}$. El sentido de un texto no se corresponde directamente con "lo que el autor quiso decir"; más bien surge como un acto de interpretación del lector. ${ }^{9}$

Esto quiere decir, como lo ha señalado la teoría literaria contemporánea, que toda obra es incompleta, que necesita del lector para ser actualizada, pero que esta actualización depende de las condiciones, los intereses y las perspectivas del lector. En este sentido, la interpretación de un texto es semejante a la interpretación de una obra musical: recrea los significados de una manera única y personal, aportando algo que la sola partitura no brinda, permitiendo el desarrollo de un estilo de lectura propio mediante el cual el lector se lee a sí mismo al interpretar la obra. Esta característica de la lectura parece más evidente en el caso de obras literarias y artísticas en las que prima lo simbólico y todo es apenas sugerido; no obstante, también en las obras filosóficas la comprensión sólo ocurre como resultado de la participación activa del lector. ¿En qué consiste esta participación?

En primer lugar, consiste en que cuando leemos realizamos una especie de traducción ${ }^{10}$ de los conceptos del texto a los conceptos que configuran nuestro propio mundo, o mejor, nuestro horizonte de comprensión. Uno carga de significado el texto a partir de significados previos, pero, a la vez, uno revisa estos significados previos, que muchas veces constituyen prejuicios e ideas vagas y ambiguas, luego los replantea a la luz del texto. En este sentido, la lectura es un ejercicio permanente de resignificación de las palabras con que acostumbramos interpretar el mundo y dirigir nuestra vida. Consecuentemente, buena parte de las dificultades que tenemos para comprender un texto surgen cuando pretendemos saber lo que significan las palabras de una vez por todas, esto es, cuando creemos tener el significado universal de los términos que nos encontramos en el texto, no permitiéndoles presentar sus nuevos matices semánticos. Por el contrario, la lectura es un ejercicio propiamente filosófico en tanto que en un modo de pensar nuestro pensamiento, de cuestionar las ideas que damos por ciertas y evidentes, de investigar aquello que sin ser pensado nos hace pensar como pensamos.

A partir de lo anterior, el lector participa en la construcción del sentido, en segundo lugar, en tanto que es necesario, como pedía Nietzsche, rumiar los textos. Esto significa habitar en ellos, plantearse personalmente sus cuestiones y seguir sus soluciones como si uno mismo las estuviera 
pensando. Pero rumiar también quiere decir permitirse pensar desde de los libros, dejarse problematizar por lo que dicen, ver a partir de lo leído, o mejor, leer como una forma de ver. Sólo así la lectura es personal y produce pasión y gozo. No se lee para aprender algo, para adquirir nuevos datos, sino para ampliar nuestra forma de ver y de vivir.

Esto nos conduce, finalmente, a que un buen lector lee pensado y dialogando con el texto. Esto es, se acerca a la obra desde sus propias preguntas y sigue sus cursos como un momento de su propia reflexión. Por un lado, interroga al texto en una búsqueda personal de la comprensión, que desborda el ejercicio de leer para el seminario, abordándolo desde sus propios problemas vitales; por el otro, se deja interrogar por el texto confrontando con él, como ya indicamos, sus propias convicciones y puntos de vista. Así, el sentido surge del encuentro dialógico entre el lector y la obra. La profundidad de este encuentro determinará, más adelante en la formación filosófica, la posibilidad de tomar distancia crítica con respecto a los autores estudiados.

Todo esto no significa, por supuesto, que la producción del significado en que consiste la lectura sea una tarea arbitraria, caprichosa y puramente subjetiva. Por el contrario, la interpretación sólo es posible si se consigue atender al texto sin violentarlo, sin imponérsele, captándolo en sus propios términos, dejándolo hablar mediante la reconstrucción de su tejido argumentativo. Pero esta atención al texto es siempre un ejercicio de encuentro con el mundo del lector, un mundo en construcción a partir de nuevas experiencias y lecturas. Esta apertura de la relación entre el lector y el texto permite que haya discusión en el seminario, el cual se convierte en un espacio para presentar, analizar y evaluar interpretaciones diversas.

\subsection{Leer, escribir y hablar como formas de cuidar}

La lectura y la escritura filosóficas son, pues, maneras de abrirse a los otros, de entrar en diálogo con los pensadores de la tradición, pero también con los compañeros del seminario. Esto sugiere que la dimensión comunitaria es fundamental en la formación filosófica. Uno no aprende filosofía nunca sólo. Y por eso, además del desarrollo de destrezas cognitivas, la formación filosófica implica el desarrollo de competencias afectivas, sociales, éticas $y$, en una palabra, la formación personal integral. Al respecto han enfatizado autores como Matthew Lipman y los promotores de Filosofía para Niños, quienes han explorado a fondo la vocación educadora de la filosofía en todos los niveles escolares y aún en escenarios extraacadémicos. ${ }^{11}$

¿En qué medida el estudio de textos contribuye a la formación personal o, más exactamente, cuáles son propiamente las dimensiones complementarias a la cognitiva que deben ser desarrolladas en la formación filosófica? En términos generales se podría responder diciendo que la formación filosófica implica la adquisición y puesta en práctica de una serie de actitudes y valores. Así, por ejemplo, la práctica del seminario requiere sin duda, además de la capacidad de realizar el estudio intrínseco de una obra, una actitud de apertura y modestia para recibir las correcciones y sugerencias de los compañeros; humildad para reconocer los propios límites y las dificultades en la comprensión y presentación de los argumentos; paciencia y tenacidad para el trabajo arduo de interpretación; valentía para expresar públicamente las propias ideas y preguntas; solidaridad para la búsqueda comunitaria de la comprensión; etc. En efecto, vicios como el engreimiento y el falso orgullo de quien descalifica a otros, suponiendo que entiende mejor los textos que ellos, resultan más nocivos para el trabajo de seminario que la falta de destreza en la lectura analítica.

No obstante, más que pensar en términos de las actitudes y los valores que debería tener un estudiante de filosofía, la 
dimensión comunitaria propia de los tipos de investigación textual que componen la disciplina filosófica de pregrado apunta hacia un elemento, que lejos de ser un añadido o un adorno es esencial al pensamiento filosófico mismo y constitutivo del filosofar. Se trata de la dimensión cuidante del pensamiento, como la ha denominado Lipman. ${ }^{12}$ Esto de la formación de un pensamiento capaz de dirigir nuestra atención hacia lo que tiene importancia para nosotros y los otros, de apreciar y valorar lo que cuenta, de comprometerse con el cultivo de lo que nos interesa, de ponernos en el lugar de otros, de guiar nuestra elección hacia aquello a lo que queremos consagrar la vida y de orientarnos en la búsqueda de lo que quisiéramos $\operatorname{ser}^{13}$. Sin duda, esto no se logra mediante el perfeccionamiento de las destrezas propias de la lectura analítica. Por el contrario, el desarrollo de estas depende justamente de qué tanto puedan los estudiantes comprometerse afectivamente con su propio proceso formativo, de qué tanto puedan amar lo que hacen, apasionarse por la lectura y la escritura.

Pero no ha de creerse que la pasión es simplemente el motor del pensamiento, que la emoción que nos indica lo que nos interesa es sólo el origen de la investigación. Antes bien, el componente afectivo es una dimensión de todo pensamiento, sin el cual la razón se instrumentaliza y se torna incapaz de dirigirse hacia la búsqueda y la construcción del sentido. Una y otra vez a lo largo de su historia, cuando la filosofía vuelve la mirada sobre sí misma, descubre que es deseo puesto en movimiento mediante el pensamiento. Por eso, aprender a pensar es también aprender a desear; y ambos se forman sólo en el encuentro con los otros -los que pensaron antes y ahora leemos, los que fueron pensados, los que piensan con nosotros-, quienes nos abren nuevas perspectivas tanto como limitan y contradicen nuestras pretensiones desmedidas; quienes nos presentan retos y cuestiones, ante quienes debemos presentar lo que somos en lo que hacemos y decimos; quienes comparten las incertidumbres, las dificultades y los gozos de la búsqueda de comprensión, para quienes nuestras tentativas de lectura y escritura son también textos que tejen la vida.

\section{Referencias bibliográficas}

Cuesta Abad, J. M. y Jiménez Heffernan, J. (2005) Teorías Literarias del siglo XX, Madrid: Akal.

Lipman, M. (1997) Pensamiento complejo y educación, Madrid: La Torre.

(2003) La educación para el pensamiento cuidadoso En: Thinking in Education, Cambridge: Cambridge University Press, Traducción inédita de Diego Pineda.

Nussbaum, M. (1997) Justicia Poética, Santiago de Chile: Andrés Bello.

Pineda, D. (enero 2005) Contenido filosófico y forma literaria, en: Novedades educativas, No. 169.

(2007) La lectura como búsqueda y construcción de significado, Bogotá: Cedinpro.

Zuleta, E. (1985) Sobre la lectura, En: Sobre la idealización en la vida personal y colectiva y otros ensayos, Bogotá: Procultura. basa en emociones con valor cognitivo, es decir, en emociones que se apoyan siempre en creencias y razones; c) es un pensamiento activo pues conduce a acciones y conductas coherentes con la emoción; d) es un pensamiento normativo porque se interesa por las posibilidades ideales de la propia conducta y en general reflexiona sobre lo que debería ser; y e) es un pensamiento empático porque nos permite situarnos en la perspectiva del otro. Cf. Ibíd. Sobre el valor cognitivo de las emociones Cf. Nussbaum, M. (1997) Emociones Racionales, en Op. Cit. 